\title{
RESISTÊNCIA DE GENÓTIPOS DE SORGO AO PULGÃO Rhopalosiphum maidis (Fitch, 1856) (Hemiptera: Aphididae): III. EFEITO NO DESENVOLVIMENTO DA PLANTA
}

\author{
Resistance of sorghum genotypes to the corn leaf aphid Rhopalosiphum maidis \\ (Fitch, 1856) (Hemiptera: Aphididae): III. Effect in plant development
}

\author{
Alysson Rodrigo Fonseca ${ }^{1}$, Ivan $\mathrm{Cruz}^{2}$, César Freire Carvalho ${ }^{3}$, Brígida Souza ${ }^{3}$
}

\begin{abstract}
RESUMO
Realizou-se este trabalho com o objetivo de estudar, em casa-de-vegetação, a tolerância como mecanismo de resistência do sorgo ao pulgão Rhopalosiphum maidis (Fitch), em experimentos conduzidos no Centro Nacional de Pesquisa de Milho e Sorgo - CNPMS/ EMBRAPA, Sete Lagoas, MG. Oito genótipos de sorgo, sendo sete de resistência conhecida ao pulgão Schizaphis graminum (Rondani) e a cultivar comercial BRS303, foram semeados em uma densidade de três sementes por vaso com capacidade para $5 \mathrm{Kg}$ de solo. Após a emergência, realizou-se um desbaste, deixando-se apenas uma planta, que foi coberta por uma gaiola cilíndrica revestida com "voil". Aos onze dias após o plantio, metade dos vasos contendo cada genótipo foi infestada com 25 pulgões adultos por planta, enquanto a outra metade foi utilizada como tratamento-testemunha, avaliando-se, semanalmente, a altura e o peso fresco e seco das plantas infestadas e não-infestadas. O delineamento experimental foi o inteiramente casualizado, em parcela subdividida, alocando-se, nas parcelas, os genótipos e, nas subparcelas, os períodos de avaliação, com 10 repetições. Observou-se que 'GR1.1.1.1.1', 'BRS303', 'IS2293', 'GSBTx399', 'TX430 (GR)' e 'TX2567' apresentaram a tolerância como mecanismo de resistência, embora essa tenha sido mais evidente nos três primeiros genótipos.
\end{abstract}

Termos para indexação: Sorghum, pulgão-do-milho, método de controle, resistência de plantas a insetos.

\section{ABSTRACT}

The objective of this work was to investigate in a greenhouse, the tolerance as a resistance mechanism of sorghum to the corn leaf aphid Rhopalosiphum maidis (Fitch) in experiments conducted in the National Corn and Sorghum Research Center of EMBRAPA, in Sete Lagoas, MG, Brazil. Eight sorghum genotypes, seven of them being known as resistant to the greenbug Schizaphis graminum (Rondani) and the commercial cultivar BRS303, were sown at a density of three seeds per pot with a capacity for $5 \mathrm{~kg}$ of soil. After emergence, a thinning was accomplished leaving only one plant, which was covered with a cylindrical cage, "voil"'-coated. Eleven days after sowing, half of the pots were infested with 25 greenbug (adult aphids) per plant, whereas the other half was utilized as control. Weekly the height and fresh and dry weight at infested and non-infested plants were observed. The experimental design was completely randomized in split plot by allocating in the plots the genotypes and in the subplots the evaluation periods with ten replicates. It was found that genotypes 'GR 1.1.1.1.1', 'BRS303', 'IS2293', 'GSBTx399', 'TX430 (GR)' and 'TX2567' presented tolerance as a resistance mechanism, though it had been more evident in the three first ones genotypes.

Index terms: Sorghum, control method, resistance of plants to insects.

(Recebido para publicação em 18 de fevereiro de 2003 e aprovado em 10 de abril de 2003)

\section{INTRODUÇÃO}

O pulgão-do-milho Rhopalosiphum maidis (Fitch, 1856) (Hemiptera: Aphididae) é encontrado em praticamente todas as regiões temperadas e tropicais do mundo, tendo como principais plantas hospedeiras o sorgo, a cevada e o milho. No Brasil, é encontrado principalmente em regiões onde se cultivam o sorgo e o milho "safrinha", causando danos econômicos (GOUSSAIN, 2001). Segundo Cruz et al. (1997), esses insetos desenvolvem-se em colônias, principalmente nos pontos de crescimento, como cartucho, pendão e gemas florais. São responsáveis pela sucção da seiva, além de proporcionar o desenvolvimento da fumagina e serem vetores de viroses, como o mosaico.

\footnotetext{
1. Engenheiro Agrônomo, MSc., Dr., Departamento de Entomologia da Universidade Federal de Lavras/ UFLA - Caixa Postal 37 - $37200-000$ - Lavras, MG.

2. Pesquisador CNPMS/EMBRAPA, Caixa Postal 151 - 35701-970 - Sete Lagoas, MG.
}

3. Professores do Departamento de Entomologia da UFLA. 
Entre os vários métodos que podem ser empregados para o controle de pragas, a utilização de cultivares resistentes tem se destacado como um método eficaz e prático (LARA, 1991). Uma planta apresenta a tolerância como mecanismo de resistência quando é menos danificada em relação a outras, sob um mesmo nível de infestação por uma determinada praga, sem afetar o comportamento ou a biologia da espécie fitófaga. Assim, pode-se dizer que é uma planta que possui a capacidade de suportar o ataque da praga, quer seja pela regeneração dos tecidos destruídos, emissão de ramos ou perfilhos, quer seja por outro meio qualquer, de forma que o ataque não chegue a provocar queda significativa na quantidade e qualidade de sua produção. Esse mecanismo de resistência depende exclusivamente da planta e não atua sobre o inseto (HORBER, 1980; TINGEY, 1986; LARA, 1991; REESE et al., 1994).

Segundo Reese et al. (1994), a tolerância apresenta algumas vantagens em relação aos mecanismos de antibiose e não-preferência. Primeiramente, pelo fato de ser uma resposta da planta, não afeta o comportamento, reprodução ou desenvolvimento do inseto, não exercendo, portanto, pressão de seleção, o que pode ocorrer com a antibiose e a não-preferência. Essa característica é de fundamental importância para o desenvolvimento de cultivares resistentes a afídeos, insetos que apresentam uma grande capacidade de desenvolverem biótipos capazes de "quebrar" um determinado mecanismo de resistência de uma planta. Uma segunda vantagem é que a tolerância não apresenta nenhum efeito deletério sobre os inimigos naturais das pragas, o que pode ocorrer com a antibiose e não-preferência. Assim, esse mecanismo de resistência pode ser importante na manutenção de populações de predadores e parasitóides, porque, além de não afetar diretamente esses organismos, não reduz a população de suas presas e hospedeiros. Outra vantagem desse mecanismo é a possibilidade de aumento do nível de dano econômico, atrasando ou reduzindo o controle químico da praga. Entretanto, segundo Teetes (1980) e Reese et al. (1994), a tolerância apresenta como desvantagem a dificuldade de separá-la da antibiose e nãopreferência e, ainda, de quantificá-la com precisão, principalmente pelo fato de ser mais afetada pela temperatura, sendo reduzida ou mesmo anulada sob condições térmicas mais baixas (WOOD JÚNIOR e STARKS, 1972; SCHWEISSING e WILDE, 1979; TINGEY e SINGH, 1980).

De acordo com Starks et al. (1983) e Webster et al. (1987, 1991), a tolerância é definida em função da produção e, portanto, é a planta, e não o inseto, que deve ser medida. Assim, uma das técnicas para quantificar e separar a tolerância da antibiose é a utilização de um número fixo de insetos, adicionando-os ou removendo-os, diariamente, de maneira a manter uma população sempre constante. Entretanto, essa técnica limita o número de plantas que podem ser observadas em um intervalo de tempo, em função da mão-de-obra envolvida (WEBSTER et al., 1991; REESE et al., 1994). Outros métodos para quantificar a tolerância a afídeos, em sorgo, incluem índices que consideram a relação entre o peso de plantas infestadas e não-infestadas, além do número de insetos obtidos no final de um período de tempo (DIXON et al., 1990; INAYATULLAH et al., 1990). Também têm sido utilizadas a diferença nos danos (STARKS et al., 1983), perda em área foliar (SCHWEISSING e WILDE, 1979) e diferença em altura entre plantas infestadas e não-infestadas (SCHUSTER e STARKS, 1973).

Assim, considerando as possibilidades de utilização da tolerância como mecanismo de resistência do sorgo a afídeos, especialmente ao pulgão $R$. maidis, objetivou-se com este trabalho determinar o efeito de populações do pulgão sobre o desenvolvimento de oito genótipos de sorgo.

\section{MATERIAL E MÉTODOS}

Os ensaios foram conduzidos em casa-devegetação no Centro Nacional de Pesquisa de Milho e Sorgo - EMBRAPA, Sete Lagoas, MG, empregando-se os genótipos BRS303; GB3; GR1.1.1.1.1; GSBTx399; IS2293; TX2567; TX430 (GR) e TX430 (IS x SC), sendo o primeiro um híbrido comercial e os demais apresentando um ou mais mecanismos de resistência ao pulgão Schizaphis graminum (Rondani, 1852), conforme Cruz (1986).

Inicialmente, determinou-se o número de pulgões necessários para provocar um dano significativo em plantas suscetíveis, conforme a metodologia proposta por Cruz (1986). O experimento para esse fim foi conduzido com o híbrido comercial BR304, plantado em 20 vasos de $20 \mathrm{~cm}$ de diâmetro e $25 \mathrm{~cm}$ de altura, com capacidade para $5 \mathrm{Kg}$ de solo, adubado com NPK na formulação 04-14-08 + Zn, na proporção de 3 $\mathrm{Kg} / 1000 \mathrm{Kg}$ de solo, conforme análise de solo.

Onze dias após o plantio, foram liberados 5, 10, 15,20 e 25 pulgões adultos e ápteros por vaso, que continha uma só planta. O tratamento/testemunha consistiu de plantas não-infestadas. Cada unidade foi coberta por 
uma gaiola cilíndrica feita com armação de arame com $20 \mathrm{~cm}$ de diâmetro e $40 \mathrm{~cm}$ de altura, envolta por um tecido fino "voil". Após a infestação, foram feitas observações diárias em cada planta, retirando-se ninfas produzidas e repondo os pulgões adultos mortos ou desaparecidos, de modo a manter a população inicial. Determinou-se a altura das plantas, considerando-se a distância entre a sua base e o ápice da folha mais longa. As avaliações foram iniciadas no dia da infestação e, posteriormente, a intervalos semanais, até a constatação de uma diferença significativa entre as parcelas não infestadas e pelo menos uma parcela infestada, conforme metodologia adaptada por Cruz (1986).

Pelos resultados obtidos, pôde-se conduzir o experimento visando a conhecer o mecanismo de resistência por tolerância nos diferentes genótipos. Para esse teste, três sementes de cada genótipo foram plantadas em vasos, como descrito anteriormente, e, após a emergência, foi realizado um desbaste deixando-se uma planta por vaso. Cada unidade foi coberta com o mesmo tipo de gaiola usada no ensaio preliminar e, onze dias após o plantio, metade dos vasos, de cada genótipo, foi infestada com a densidade de pulgões escolhida com base nos resultados do experimento inicial, ou seja, 25 pulgões/planta, enquanto na outra metade, considerada como testemunha, não foi feita liberação, permitindo-se comparar o desenvolvimento da planta na presença e ausência do afídeo. De forma semelhante e com o mesmo objetivo do experimento preliminar, procedeuse à retirada de ninfas e à reposição de adultos. A avaliação da altura das plantas foi semelhante à descrita anteriormente, sendo a última realizada aos 21 dias após a liberação. Calculou-se o crescimento percentual das plantas, computando-se o valor do acréscimo no comprimento de cada genótipo infestado em relação ao acréscimo médio no comprimento das plantas nãoinfestadas.

Com o objetivo de se comparar o peso fresco e seco de genótipos infestados e não-infestados, após a última avaliação, realizada aos 21 dias, as plantas foram cortadas rente ao solo e acondicionadas individualmente em sacos de papel, levadas para o laboratório e imediatamente pesadas, a fim de se obter o peso fresco. Logo após, os genótipos foram colocados em estufa a $70^{\circ} \mathrm{C}$ e mantidos por um período de 72 horas, sendo novamente pesados, obtendo-se o peso seco.

As condições de temperatura e umidade relativa durante os ensaios foram registradas por um termohigrógrafo colocado próximo ao experimento e em local protegido da incidência direta de raios solares. As determinações médias diárias da temperatura e umidade relativa do ar foram feitas empregando-se a metodologia citada em Climanálise (1998), pelas fórmulas:

$$
\begin{gathered}
\mathrm{T}_{\text {média }}=\underline{\mathrm{T}}_{9}+\mathrm{T}_{\underline{\mathrm{m}}}+\frac{\mathrm{T}_{\mathrm{x}}+2 \mathrm{~T}_{21}}{5} \\
\mathrm{UR}_{\text {média }}=\underline{\mathrm{UR}_{9}} \underline{+\mathrm{UR}_{15}} \underline{+2 \mathrm{UR}_{21}}
\end{gathered}
$$

sendo:

$\mathrm{T}_{\text {média }}=$ temperatura média em ${ }^{\circ} \mathrm{C}$

$\mathrm{T}_{9}=$ temperatura às 9 horas

$\mathrm{T}_{\mathrm{m}}=$ temperatura mínima

$\mathrm{T}_{\mathrm{x}}=$ temperatura máxima

$\mathrm{T}_{21}=$ temperatura às 21 horas

$\mathrm{UR}_{\text {média }}=$ umidade relativa média em \%

$\mathrm{UR}_{9}=$ umidade relativa às 9 horas

$\mathrm{UR}_{15}=$ umidade relativa às 15 horas

$\mathrm{UR}_{21}=$ umidade relativa às 21 horas

O fotoperíodo não foi controlado, entretanto, segundo o Setor de Climatologia da EMBRAPA/ CNPMS, na época e local dos estudos, foi de aproximadamente 14 horas de luz. O delineamento experimental foi inteiramente casualizado, sob esquema de parcela subdividida, alocando-se nas parcelas os genótipos e nas subparcelas os tempos de avaliação, com dez repetições. Os dados foram submetidos à análise de variância e as médias, comparadas pelo teste de Scott e Knott a 5\% de probabilidade (SCOTT e KNOTT, 1974).

\section{RESULTADOS E DISCUSSÃO}

Observou-se que, de um modo geral, o incremento na altura das plantas foi progressivamente reduzido de acordo com o aumento na densidade de insetos liberados, variando de $29,6 \mathrm{~cm}$ em plantas não infestadas a $23,5 \mathrm{~cm}$ na densidade de 25 pulgões, na qual ocorreu uma redução percentual de 20,6\% em relação à testemunha (Tabela 1).

Pelos resultados referentes ao crescimento percentual de cada genótipo em relação a plantas nãoinfestadas, evidenciou-se, na primeira avaliação realizada no sétimo dia após a infestação, que os genótipos mais tolerantes foram o GR1.1.1.1.1, GSBTx399, IS2293 e TX430 (GR), os quais diferiram significativamente dos demais, com crescimentos percentuais de 
89,$8 ; 67,9 ; 72,1$ e 78,8\%, respectivamente (Tabela 2). Na segunda avaliação, aos 14 dias após a infestação, os genótipos GR1.1.1.1.1 e IS2293 apresentaram um crescimento percentual de cerca de 83,3 e $83,5 \%$, respectivamente. Os genótipos BRS303, GSBTx399, TX430 (GR) e TX2567 mostraram crescimentos intermediários, sendo as menores taxas obtidas para o TX430 (IS x SC) e GB3, correspondendo a 32,6 e $22,1 \%$, respectivamente. Na última avaliação, realizada aos 21 dias após a infestação, esses dois genótipos foram os únicos a se diferenciarem dos demais, com crescimento médio de 34,4 e $22,9 \%$, respectivamente. Dessa forma, um desenvolvimento mais próximo ao verificado em plantas não-infestadas foi observado nos genótipos GR1.1.1.1.1, BRS303, GSBTx399, IS2293, TX430 (GR) e TX2567, estando o mecanismo de tolerância provavelmente presente nesses materiais.

Cruz (1986) e Cruz e Vendramim (1998), estudando em casa-de-vegetação a resistência do sorgo ao pulgão $S$. graminum, verificaram que os genótipos TX2567, GR1.1.1.1.1, TX 430 (GR) e GB3 apresentaram tolerância a esse inseto. Esses resultados foram semelhantes aos obtidos neste trabalho, com exceção do genótipo GB3, que se mostrou suscetível.

O híbrido comercial BRS303, estando entre os genótipos que mostraram os menores percentuais de crescimento na primeira avaliação, recuperou-se até ficar entre aqueles que apresentaram as maiores porcentagens, principalmente na última avaliação. Esse comportamento mostra uma maior susceptibilidade da planta ao inseto nos primeiros sete dias de seu desenvolvimento e uma maior capacidade de recuperação nos estádios posteriores. Os genótipos GR1.1.1.1.1, GSBx399, IS2293, TX2567 e GB3 mantiveram uma taxa de crescimento constante nos diferentes períodos de avaliação.

Embora no genótipo TX430 (GR) a taxa de crescimento tenha sido reduzida significativamente, na avaliação realizada aos 14 dias após a infestação observou-se uma recuperação na avaliação realizada aos 21 dias, com 70,2\%, ficando entre os mais tolerantes ao inseto. O genótipo TX430 (IS x SC) mostrou uma redução gradativa de seu crescimento percentual a partir da primeira avaliação, evidenciando susceptibilidade ao afídeo durante o período avaliado.

TABELA 1 - Incremento $^{1}$ na altura $( \pm \text { EP })^{2}$ da cultivar de sorgo BR304, sete dias após infestação por adultos de Rhopalosiphum maidis em diferentes densidades. Temperatura de 28,5 $\pm 9,8^{\circ} \mathrm{C}$, UR de $78 \pm 14 \%$. CNPMS, Sete Lagoas - MG, 2001.

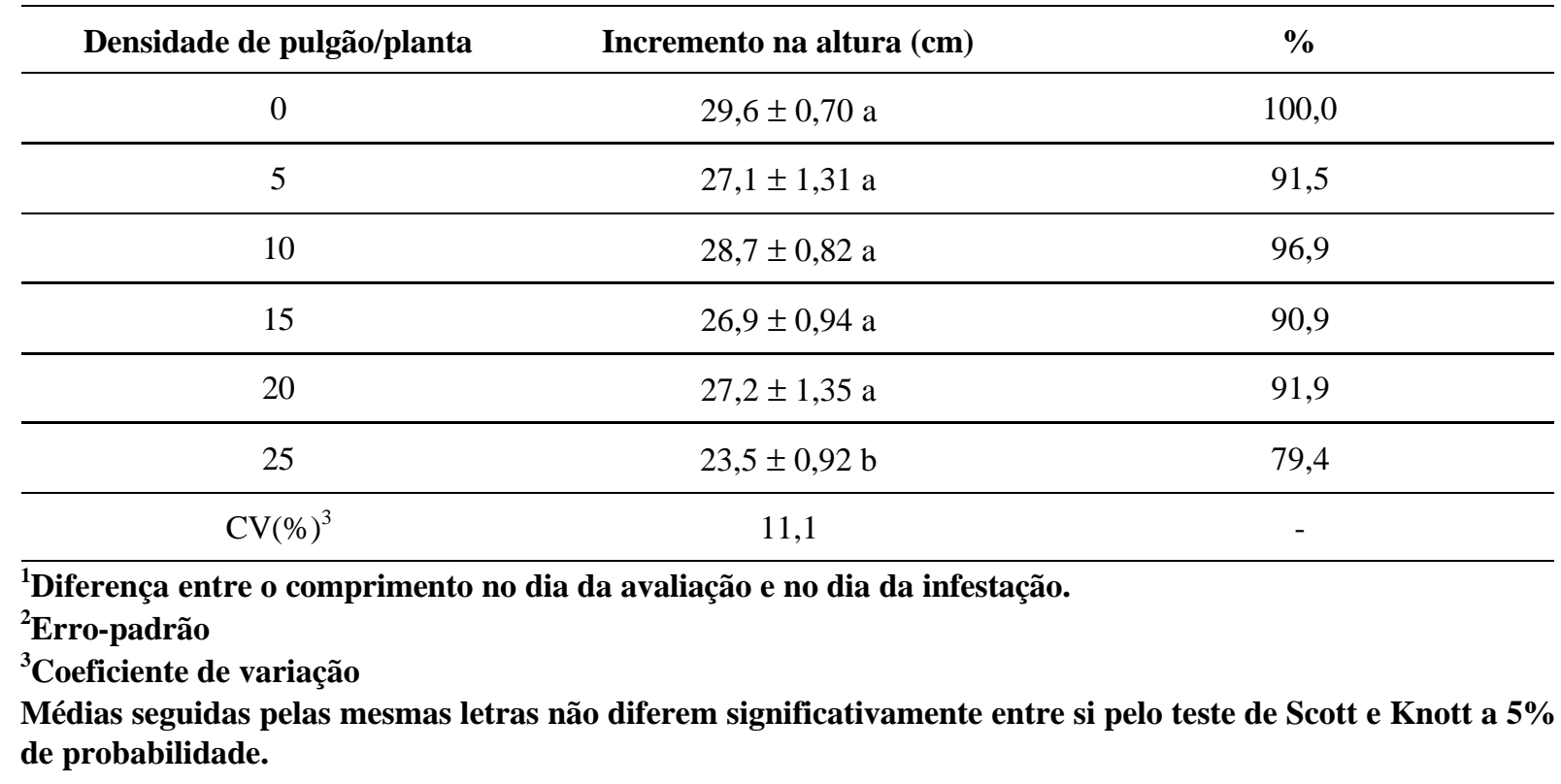

Ciênc. agrotec., Lavras, v. 28, n. 3, p. 585-592, maio/jun., 2004 
TABELA 2 - Crescimento percentual $( \pm \mathrm{EP})^{1}$ de genótipos de sorgo infestados com 25 adultos de Rhopalosiphum maidis, em relação a plantas não-infestadas, em função do tempo de avaliação. Temperatura de $27,9 \pm 7,7^{\circ} \mathrm{C}, \mathrm{UR}$ de $84 \pm 12 \%$. CNPMS, Sete Lagoas - MG, 2001 .

\begin{tabular}{lccc}
\hline \multirow{2}{*}{ Genótipos } & \multicolumn{3}{c}{ Período de avaliação (dias) } \\
\cline { 2 - 4 } & $\mathbf{7}$ & $\mathbf{1 4}$ & $\mathbf{2 1}$ \\
\hline GR1.1.1.1.1 & $89,8 \pm 4,82 \mathrm{aA}$ & $83,3 \pm 9,79 \mathrm{aA}$ & $82,1 \pm 10,69 \mathrm{aA}$ \\
\hline BRS303 & $37,5 \pm 8,11 \mathrm{bB}$ & $51,2 \pm 8,84 \mathrm{bB}$ & $74,6 \pm 11,23 \mathrm{aA}$ \\
\hline GSBTx399 & $67,9 \pm 8,39 \mathrm{aA}$ & $57,2 \pm 7,43 \mathrm{bA}$ & $71,8 \pm 8,84 \mathrm{aA}$ \\
\hline IS2293 & $72,1 \pm 9,56 \mathrm{aA}$ & $83,5 \pm 6,95 \mathrm{aA}$ & $71,6 \pm 10,26 \mathrm{aA}$ \\
\hline TX430 (GR) & $78,8 \pm 8,48 \mathrm{aA}$ & $42,6 \pm 12,46 \mathrm{bB}$ & $70,2 \pm 19,77 \mathrm{aA}$ \\
\hline TX2567 & $59,0 \pm 6,88 \mathrm{bA}$ & $45,2 \pm 11,37 \mathrm{bA}$ & $58,7 \pm 5,71 \mathrm{aA}$ \\
\hline TX430 (IS x SC) & $56,0 \pm 9,63 \mathrm{bA}$ & $32,6 \pm 7,88 \mathrm{cB}$ & $34,4 \pm 9,89 \mathrm{bB}$ \\
\hline GB3 & $38,0 \pm 6,64 \mathrm{bA}$ & $22,1 \pm 6,92 \mathrm{cA}$ & $22,9 \pm 8,15 \mathrm{bA}$ \\
\hline CV $(\%)^{2}$ & 39,7 & 52,4 & 56,3 \\
\hline
\end{tabular}

\section{${ }^{1}$ Erro-padrão}

${ }^{2}$ Coeficiente de variação

Médias seguidas pela mesma letra minúscula, nas colunas, e maiúscula, nas linhas, não diferem significativamente entre si pelo teste de Scott e Knott a $5 \%$ de probabilidade.

Observou-se que o peso fresco de plantas infestadas e não-infestadas diferiu significativamente em todos os genótipos estudados, mostrando que, embora alguns possam ter sido mais tolerantes, todos foram afetados negativamente pelo pulgão quando submetidos a uma densidade de 25 insetos/planta (Figura 1). Os pesos obtidos para plantas dos genótipos GR1.1.1.1.1; BRS303 e IS2293 infestadas com $R$. maidis foram os que mais aproximaram-se da testemunha, mostrando ser os mais tolerantes ao pulgão. Esses resultados estão de acordo com os apresentados na Tabela 2, na qual se evidencia que esses materiais estão entre os que apresentaram maior crescimento percentual. As maiores diferenças entre o peso fresco de plantas infestadas e a testemunha foram observadas para os genótipos TX430 (IS x SC) e GB3, mostrando ser os mais afetados pela presença do pulgão, reduzindo a produção de matéria verde e, conseqüentemente, o peso.

De uma maneira geral, pelos resultados obtidos para o peso seco de plantas infestadas e nãoinfestadas, evidencia-se uma resposta semelhante à obtida para o peso fresco (Figura 2). Entretanto, observouse que o genótipo GR1.1.1.1.1 não mostrou diferença significativa entre o peso seco de plantas infestadas e não-infestadas, sendo de 2,6 e 3,6 g, respectivamente, evidenciando ser, entre os genótipos estudados, o que menos teve a produção de matéria verde reduzida pelo ataque do pulgão $R$. maidis.

Em razão do crescimento percentual, os genótipos GR1.1.1.1.1, BRS303, IS2293, GSBTx399, TX430 (GR) e TX2567 apresentaram tolerância como mecanismo de resistência, embora essa tenha sido mais evidente para o primeiro. 


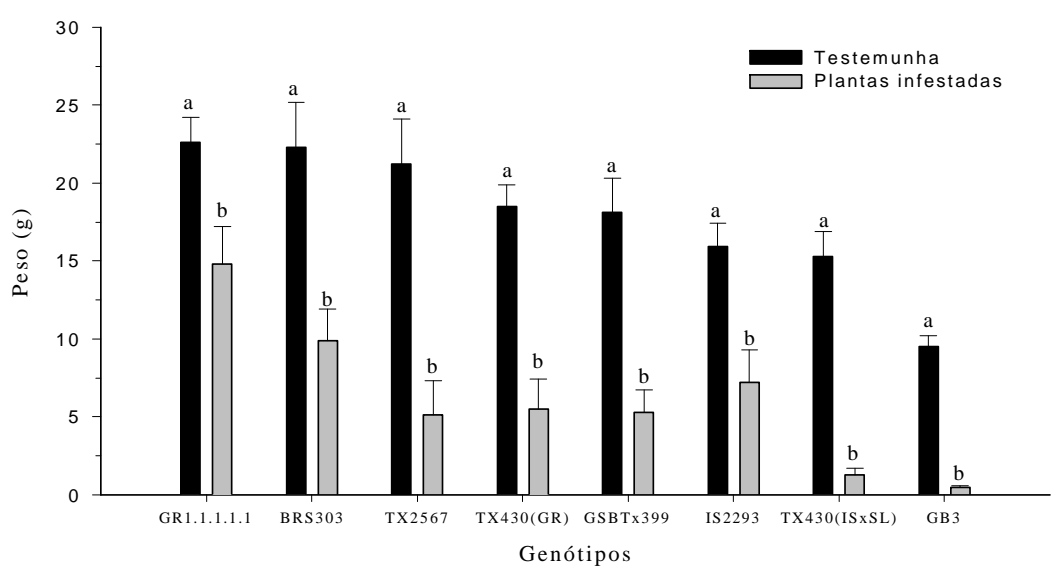

FIGURA 1 - Peso fresco ( \pm EP) de genótipos de sorgo infestados com 25 adultos de Rhopalosiphum maidis em relação a plantas não-infestadas, aos 21 dias após a infestação. Médias dos tratamentos com diferenças significativas pelo teste de Scott e Knott a 5\% de probabilidade. CNPMS, Sete Lagoas, 2001.

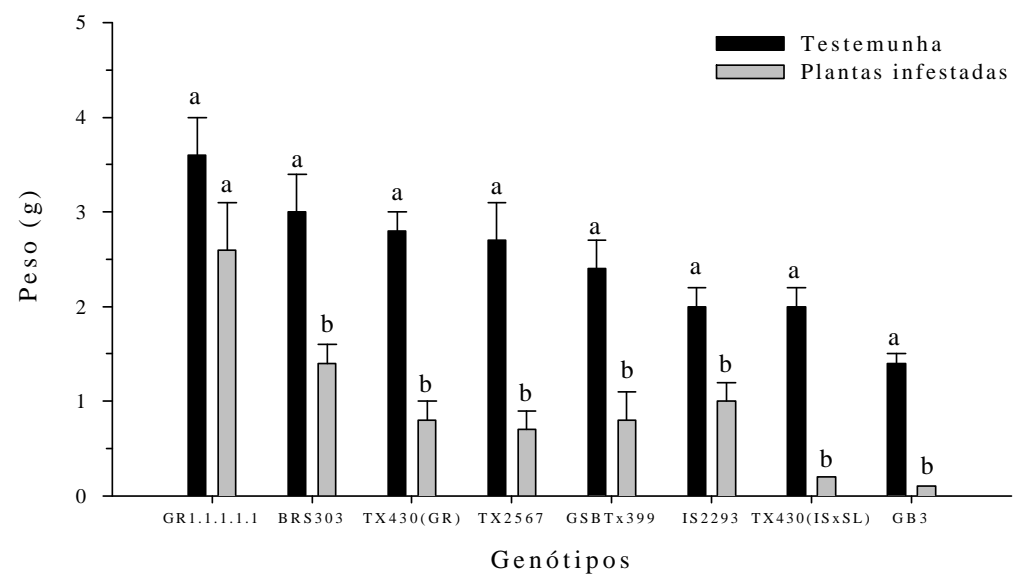

FIGURA 2 - Peso seco ( \pm EP) de genótipos de sorgo infestados com 25 adultos de Rhopalosiphum maidis em relação a plantas não-infestadas, aos 21 dias após a infestação. Médias dos tratamentos com diferenças significativas pelo teste de Scott e Knott a 5\% de probabilidade. CNPMS, Sete Lagoas, 2001. 


\section{CONCLUSÕES}

a) O pulgão Rhopalosiphum maidis afetou o crescimento e o peso dos genótipos de sorgo estudados.

b) O genótipo GR1.1.1.1.1 foi tolerante ao pulgão Rhopalosiphum maidis e os demais, BRS303, IS2293, GSBTx399, TX430 (GR) e TX2567, apresentaram menor nível de tolerância.

c) Os genótipos GB3 e TX430 (IS x SC) foram os mais suscetíveis ao pulgão, com base no crescimento percentual e produção de biomassa.

\section{REFERÊNCIAS BIBLIOGRÁFICAS}

CLIMANÁLISE. Boletim de monitoramento e análise climática. Cachoeira Paulista, 1998. 45 p.

CRUZ, I. Resistência de genótipos de sorgo ao pulgão-verde Schizaphis gramimun (Rondani, 1852) (Homoptera: Aphididae). 1986. 222 f. Tese (Doutorado em Entomologia) - Escola Superior de Agricultura Luiz de Queiroz, Piracicaba, 1986.

CRUZ, I.; VALICENTE, F. H.; SANTOS, J. P. dos; WAQUIL, J. M.; VIANA, P. A. Manual de identificação de pragas da cultura do milho. Sete Lagoas: EMBRAPA/CNPMS, 1997. 67 p.

CRUZ, I.; VENDRAMIM, J. D. Tolerância como mecanismo de resistência de sorgo ao pulgão-verde, Schizaphis graminum (Rond.) (Homoptera: Aphididae). Anais da Sociedade Entomológica do Brasil, Curitiba, v. 27, n. 1, p. 141-148, mar. 1998.

DIXON, A. G. O.; BRAMEL-COX, P. J.; REEVE, J. C.; HARVEY, T. L. Mechanisms of resistance and their interactions in twelve sources of resistance to biotype $\mathrm{E}$ greenbug (Homoptera: Aphididae) in sorghum. Journal of Economic Entomology, Lanham, v. 83, n. 1, p. 234-240, Feb. 1990.

GOUSSAIN, M. M. Efeito da aplicação do silício em plantas de milho no desenvolvimento biológico da lagarta-do-cartucho Spodoptera frugiperda (J. E. Smith, 1797) e do pulgão-da-folha Rhopalosiphum maidis (Fitch, 1856) (Hemiptera: Aphididae). 2001. 64 p. Dissertação (Mestrado em Entomologia) - Universidade Federal de Lavras, Lavras, 2001.

INAYATULLAH, C.; WEBSTER, J. A.; FARGO, W. $\mathrm{S}$. Index measuring plant resistance to insects.
Entomologist, London, v. 109, n. 3, p. 146-152, Sept. 1990.

LARA, F. M. Princípios de resistência de plantas a insetos. São Paulo: Ícone, 1991. 336 p.

HORBER, E. Types and classification of resistance. In: FOWDEN, F. G.; JENNINGS, P. R. (Ed.). Breeding plants resistant to insects. New York: John Wiley \& Sons, 1980. p. 87-113.

REESE, J. C.; SCHWENKE, J. R.; LAMONT, P. S.; ZEHR, D. D. Importance and quantification of plant tolerance in crop pest management programs for aphids: greenbug resistance in sorghum. Journal of Agricultural Entomology, Clemson, v. 11, n. 3, p. 255-270, July 1994.

SCHUSTER, D. J.; STARKS, K. J. Greenbugs: components of host-plant resistance in sorghum. Journal of Economic Entomology, Lanham, v. 66, n. 5, p. 1131-1134, Oct. 1973.

SCHWEISSING, F. C.; WILDE, G. Temperature and plant nutrient effects on resistance of seedling sorghum to the greenbug. Journal of Economic Entomology, Lanham, v. 72, n. 1, p. 20-23, 1979.

SCOTT, A.J.; KNOTT, M.A.A cluster analysis method for grouping means in the analysis of variance. Biometrics, Washington, v. 30, n. 3, p. 507-512, Sept. 1974.

STARKS, K. J.; BURTON, R. L.; MERKLE, O. G. Greenbugs (Homoptera: Aphididae) plant resistance in small grain and sorghum to biotype E. Journal of Economic Entomology, Lanham, v. 76, n. 4, p. 877$880,1983$.

TEETES, G. L. Breeding sorghum resistant to insects. In: MAXWELL, F. G.; JENNINGS, P. R. (Eds.). Breeding plants resistant to insects. New York: [s.n.], 1980. 683 p.

TINGEY, W. M. Techniques for evaluating plant resistance to insects. In: MILLER, J. R.; MILLER, T. A. (Eds.). Insect-plant interactions. New York: Springer-Verlag, 1986. p. 251-284.

TINGEY, W. M.; SINGH, S. R. Environmental factors influencing the magnitude and expression of resistance. In: FOWDEN, F. G.; JENNINGS, P. R. (Eds.). Breeding plants resistant to insects. New York: John Wiley \& Sons, 1980. p. 87-113. 
WEBSTER, J. A.; BAKER, C. A.; PORTER, D. R. Detection and mechanisms of russian wheat aphid (Homoptera: Aphididae) resistance in barley. Journal of Economic Entomology, Lanham, v. 84, n. 2, p. 669667, Apr. 1991.

WEBSTER, J. A.; STARKS, K. J.; BURTON, R. L. Plant resistance studies with Diuraphis noxia (Homoptera:
Aphididae), a new United States wheat pest. Journal of Economic Entomology, Lanham, v. 80, n. 4, p. 944949, 1987.

WOOD Jr., E. A.; STARKS, K. J. Effect of temperature and host plant interaction on the biology of three biotypes of the greenbug. Environmental Entomology, Lanham, v. 1, n. 2, p. 230-234, Apr. 1972. 